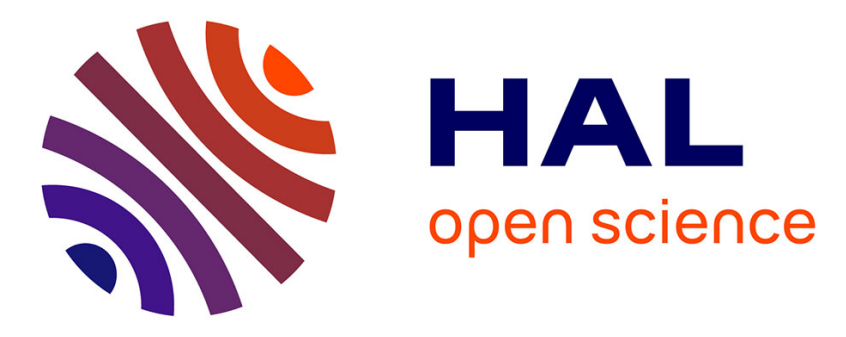

\title{
Roll Invariant Target Detection based on PolSAR Clutter Models
}

Lionel Bombrun, Gabriel Vasile, Michel Gay, Jean-Philippe Ovarlez, Frédéric

Pascal

\section{- To cite this version:}

Lionel Bombrun, Gabriel Vasile, Michel Gay, Jean-Philippe Ovarlez, Frédéric Pascal. Roll Invariant Target Detection based on PolSAR Clutter Models. IGARSS 2010 - IEEE International Geoscience and Remote Sensing Symposium, Jul 2010, Honolulu, Hawaii, United States. pp.4, 10.1109/IGARSS.2010.5653522 . hal-00520566

\section{HAL Id: hal-00520566 https://hal.science/hal-00520566}

Submitted on 23 Sep 2010

HAL is a multi-disciplinary open access archive for the deposit and dissemination of scientific research documents, whether they are published or not. The documents may come from teaching and research institutions in France or abroad, or from public or private research centers.
L'archive ouverte pluridisciplinaire HAL, est destinée au dépôt et à la diffusion de documents scientifiques de niveau recherche, publiés ou non, émanant des établissements d'enseignement et de recherche français ou étrangers, des laboratoires publics ou privés. 


\title{
ROLL INVARIANT TARGET DETECTION BASED ON POLSAR CLUTTER MODELS
}

\author{
Lionel Bombrun ${ }^{1,2}$, Gabriel Vasile ${ }^{1}$, Michel Gay ${ }^{1}$, Jean-Philippe Ovarlez $z^{2,3}$ and Frédéric Pascal ${ }^{2}$ \\ ${ }^{1}$ : Grenoble-Image-sPeech-Signal-Automatics Lab, CNRS / Grenoble-INP, FRANCE, \\ \{lionel.bombrun|gabriel.vasile|michel.gay\}@gipsa-lab.grenoble-inp.fr \\ 2 : SONDRA, Supélec, FRANCE, frederic.pascal@supelec.fr \\ ${ }^{3}$ : French Aerospace Lab, ONERA DEMR/TSI, FRANCE, ovarlez@onera.fr
}

\begin{abstract}
Based on the Kennaugh-Huynen decomposition, the Target Scattering Vector Model (TSVM) allows to extract four rollinvariant parameters. Those parameters are necessary for an unambiguous description of the target scattering mechanism. The proposed method consists in applying the TSVM prior to the GLRT-LQ detector for the detection of any oriented target.
\end{abstract}

Index Terms - Polarimetric Synthetic Aperture Radar, Roll-invariant decomposition, Target detection.

\section{INTRODUCTION}

In this paper, a method is proposed for detecting Polarimetric Synthetic Aperture Radar (PolSAR) targets. The proposed method is a combination of the Target Scattering Vector Model (TSVM) and the Generalized Likelihood Ratio Test Linear Quadratic (GLRT-LQ) detector. The TSVM provides an unique and roll-invariant decomposition of the observed target vector by means of four independent parameters. The combination of those two methods will allow the detection of any oriented targets (trihedral, dihedral, dipole, helix, ...).

This paper is organized as follows. The context of the study is first described. Then, the TSVM algorithm is exposed. Next, the proposed algorithm for a roll-invariant target detection is presented. Then, some detection results are shown on a real PolSAR data-set acquired by the RAMSES sensor at X-band.

\section{ROLL-INVARIANT TARGET DECOMPOSITION}

\subsection{Context}

Let $\mathbf{k}_{d i p}$ and $\mathbf{k}_{d i h}$ be respectively the steering vectors in the Pauli basis of two oriented dipole and dihedral. They are respectively defined by:

$$
\mathbf{k}_{d i p}=\frac{1}{\sqrt{2}}\left[\begin{array}{c}
1 \\
\cos (2 \psi) \\
\sin (2 \psi)
\end{array}\right] \text { and } \mathbf{k}_{d i h}=\left[\begin{array}{c}
0 \\
\cos (2 \psi) \\
\sin (2 \psi)
\end{array}\right]
$$

where $\psi$ is the orientation of the maximum polarization with respect to the horizontal polarization [1].

Consequently, for a roll-invariant target dipole or dihedral detection, the tilt angle $\psi$ should be removed. In 1993 , Krogager has proposed an algorithm to derive $\psi$ which uses the phase difference between right-right $\left(S_{R R}\right)$ and left-left $\left(S_{L L}\right)$ circular polarizations of the scattering matrix $\mathbf{S}$ [2]:

$$
\psi_{\text {Krogager }}=\left[\operatorname{Arg}\left(S_{R R} S_{L L}^{*}+\pi\right)\right] / 4 .
$$

where $S_{R R}$ and $S_{L L}$ are respectively defined by:

$$
\left\{\begin{array}{l}
S_{R R}=\left(S_{H H}-S_{V V}+2 j S_{H V}\right) / 2 \\
S_{L L}=\left(S_{V V}-S_{H H}+2 j S_{H V}\right) / 2
\end{array}\right.
$$

This estimated orientation angle $\psi_{\text {Krogager }}$ is valid under certain condition on the target. To overcome this problem, authors propose to apply the TSVM method which provides an unique and roll-invariant decomposition of any targets [1].

\subsection{The Kennaugh-Huynen con-diagonalization}

In PolSAR imagery, coherent targets are fully described by their scattering matrix $\mathbf{S}$. To retrieve parameters with a physical meaning, Kennaugh and Huynen have proposed to apply the characteristic decomposition on the scattering matrix [3] [4] [5]. Under the reciprocity assumption, the cross-polarization terms $S_{H V}$ ans $S_{V H}$ are equal. It yields:

$$
\mathbf{S}=\mathbf{R}(\psi) \mathbf{T}\left(\tau_{m}\right) \mathbf{S}_{\mathbf{d}} \mathbf{T}\left(\tau_{m}\right) \mathbf{R}(-\psi),
$$

where $\mathbf{R}(\psi)$ and $\mathbf{T}\left(\tau_{m}\right)$ are defined by :

$$
\mathbf{R}(\psi)=\left[\begin{array}{cc}
\cos \psi & -\sin \psi \\
\sin \psi & \cos \psi
\end{array}\right]
$$

and:

$$
\mathbf{T}\left(\tau_{m}\right)=\left[\begin{array}{cc}
\cos \tau_{m} & -j \sin \tau_{m} \\
-j \sin \tau_{m} & \cos \tau_{m}
\end{array}\right] .
$$

$\mathbf{S}_{\mathbf{d}}$ is a diagonal matrix which contains the two complex coneigenvalues $\mu_{1}$ and $\mu_{2}$ of $\mathbf{S}$ as:

$$
\mathbf{S}_{\mathbf{d}}=\left[\begin{array}{cc}
m e^{2 j(\nu+\rho)} & 0 \\
0 & m \tan ^{2} \gamma e^{-2 j(\nu-\rho)}
\end{array}\right]=\left[\begin{array}{cc}
\mu_{1} & 0 \\
0 & \mu_{2}
\end{array}\right] .
$$


The Kennaugh-Huynen con-diagonalization allows to characterize a coherent target by means of six independent parameters : $\psi, \tau_{m}, m, \gamma, \nu$ and $\rho . \psi$ is the rotation angle (see (1)). This parameter is used for the subtraction of the target orientation from the target vector, which leads to a roll-invariant decomposition. This step is named desying. $\tau_{m}$ is the target helicity, it characterizes the symmetry of the target. $m$ is the maximum amplitude return. $\gamma$ and $\nu$ are respectively the characteristic and skip angles. $\rho$ is the absolute phase of the target. This term is not observable except for interferometric applications.

\subsection{The Target Scattering Vector Model}

In 2007, Touzi has proposed a new model: the Target Scattering Vector Model (TSVM). It consists in the projection in the Pauli basis of the scattering matrix con-diagonalized by the Takagi method [1]. It leads:

$$
\begin{aligned}
\overrightarrow{e_{T}} \mathbf{S V} & =m e^{j \Phi_{s}}\left[\begin{array}{ccc}
1 & 0 & 0 \\
0 & \cos (2 \psi) & -\sin (2 \psi) \\
0 & \sin (2 \psi) & \cos (2 \psi)
\end{array}\right] \\
& \times\left[\begin{array}{c}
\cos \alpha_{s} \cos \left(2 \tau_{m}\right) \\
\sin \alpha_{s} e^{j \Phi_{\alpha_{s}}} \\
-j \cos \alpha_{s} \sin \left(2 \tau_{m}\right)
\end{array}\right] .
\end{aligned}
$$

$\alpha_{s}$ and $\Phi_{\alpha_{s}}$ are the symmetric scattering type magnitude and phase. They are derived from the con-eigenvalues $\mu_{1}$ and $\mu_{2}$ of the scattering matrix $\mathbf{S}$ by:

$$
\tan \left(\alpha_{s}\right) e^{j \Phi_{\alpha_{s}}}=\frac{\mu_{1}-\mu_{2}}{\mu_{1}+\mu_{2}} .
$$

The symmetric scattering type magnitude $\alpha_{s}$ reduces to the so-called $\alpha$ parameter issued from the Cloude-Pottier decomposition for a symmetric scatterer (i.e. $\tau_{m}=0$ ).

Due to the con-eigenvalue phase ambiguity, the KennaughHuynen decomposition is not unique. Huynen's orientation angle $\psi$ should be re-evaluated. To remove this ambiguity, the following relation is applied to restrict the domain definition of $\psi$ to the interval $[-\pi / 4, \pi / 4]$ :

$$
\begin{aligned}
& \overrightarrow{e_{T}} \mathbf{S V}\left(\Phi_{s}, \psi, \tau_{m}, m, \alpha_{s}, \Phi_{\alpha_{s}}\right) \\
& \quad=\overrightarrow{e_{T}} \mathbf{S V}\left(\Phi_{s}, \psi \pm \frac{\pi}{2},-\tau_{m}, m,-\alpha_{s}, \Phi_{\alpha_{s}}\right) .
\end{aligned}
$$

As the last term of (7) is independent of the target orientation angle, it yields that the four parameters $m, \alpha_{s}, \Phi_{\alpha_{s}}$ and $\tau_{m}$ are roll-invariant. In the following, the TSVM method is first applied on the original PolSAR data-set to provide a roll-invariant target vector. To compute the target orientation angle with the TSVM decomposition, the following relation is implemented [5] [6] [7]:

$\psi_{T S V M}=\frac{1}{2} \operatorname{Arctan}\left(\frac{2 \Re \mathrm{e}\left\{\left(S_{H H}^{*}+S_{V V}^{*}\right) S_{H V}\right\}}{\Re \mathrm{e}\left\{\left(S_{H H}^{*}+S_{V V}^{*}\right)\left(S_{H H}-S_{V V}\right)\right\}}\right)$.

\subsection{Comparison between $\psi_{T S V M}$ and $\psi_{K \text { rogager }}$}

According to the TSVM, the following relation between the orientation angle $\psi_{T S V M}$ estimated by the TSVM method and $\psi_{\text {Krogager }}$ estimated with the phase difference between right-right and left-left circular polarizations can be proved [8]:

$$
\begin{aligned}
\psi_{T S V M} & =\psi_{\text {Krogager }}-\frac{1}{4} \operatorname{Arctan}\left(\frac{\tan \left(\alpha_{s}\right) \sin \left(\Phi_{\alpha_{s}}\right)}{\tan \left(\alpha_{s}\right) \cos \left(\Phi_{\alpha_{s}}\right)+\sin \left(2 \tau_{m}\right)}\right) \\
& +\frac{1}{4} \operatorname{Arctan}\left(\frac{\tan \left(\alpha_{s}\right) \sin \left(\Phi_{\alpha_{s}}\right)}{\tan \left(\alpha_{s}\right) \cos \left(\Phi_{\alpha_{s}}\right)-\sin \left(2 \tau_{m}\right)}\right) .
\end{aligned}
$$

Fig. 1 shows a comparison between the orientation angle $\psi_{T S V M}$ estimated via the TSVM and $\psi_{\text {Krogager }}$ as a function of three roll-invariant TSVM parameters: $\tau_{m}, \Phi_{\alpha_{s}}$ and $\alpha_{s}$. Fig. 1(a) shows the evolution of $\psi_{T S V M}$ and $\psi_{\text {Krogager }}$ with the helicity $\tau_{m}$ for $\alpha_{s}=\pi / 3$ and $\Phi_{\alpha_{s}}=\pi / 3$. Fig. 1(b) and Fig. 1(c) show respectively this relation as a function of the target scattering phase $\Phi_{\alpha_{s}}$ for $\alpha_{s}=\pi / 3$ and $\tau_{m}=\pi / 8$, and as a function of $\alpha_{s}$ for $\Phi_{\alpha_{s}}=\pi / 3$ and $\tau_{m}=\pi / 8$. For $\tau_{m}=0$, the target is symmetric. It leads that $\psi_{T S V M}$ is equal to $\psi_{\text {Krogager }}$, as observed in black in Fig. 1(a). Moreover, for a null target scattering phase $\Phi_{\alpha_{s}}, \psi_{K \text { rogager }}$ and $\psi_{T S V M}$ are equal. Similar observations can be done for $\alpha_{s}=0$ and $\alpha_{s}=\pi / 2$ as shown in Fig. 1(c)

For $\tau_{m}=0, \Phi_{\alpha_{s}}=0, \alpha_{s}=0$ or $\alpha_{s}=\pi / 2$, the orientation angle estimated by the phase difference between rightright and left-left circular polarizations is equal to this estimated by the TSVM. It leads that both tilt angles are equal for a wide class of targets including trihedral, dihedral, helix, dipole, quarter wave, ... For all other cases, the orientation angle $\psi_{K r o g a g e r}$ is biased, and $\psi_{T S V M}$ should be used instead for a roll-invariant target characterization.

\section{ROLL-INVARIANT TARGET DETECTION}

The general principle of the proposed roll-invariant target detection algorithm can be divided into five steps. First, the orientation angle $\psi$ is computed and the "roll-invariant" target vector is extracted. This step is named desying. Then, the covariance matrix $[M]$ of the clutter is estimated. Next, the similarity measure between the steering vector and the "rollinvariant" target vector is computed. The false alarm probability is fixed, and finally we conclude or not on the detection.

\subsection{Binary hypothesis test}

The target detection problem can be formulated as a binary hypothesis test shown in (12). Under the null hypothesis $H_{0}$, the observed target vector $\mathbf{k}$ is only the clutter $\mathbf{c}$. Under the alternative hypothesis $H_{1}$, the backscattered signal can be decomposed as the sum of the reference signal $\mathbf{p}$ times an unknown scalar complex parameter $\alpha$ with the clutter c. Here, the clutter is modeled as a Spherically Invariant Random Vector (SIRV), i.e. $\mathbf{c}=\sqrt{\tau} \mathbf{z} . \mathbf{c}$ is defined as the product of a 


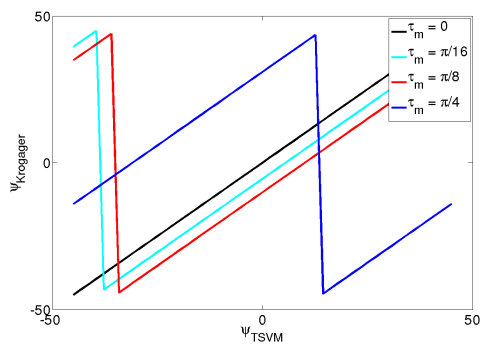

(a)

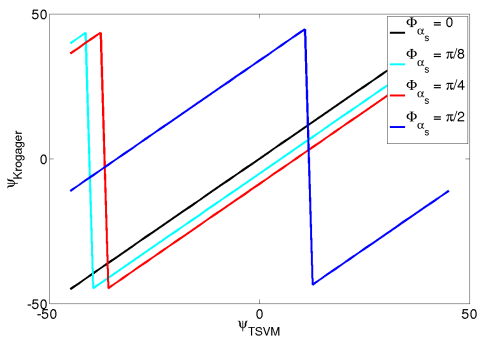

(b)

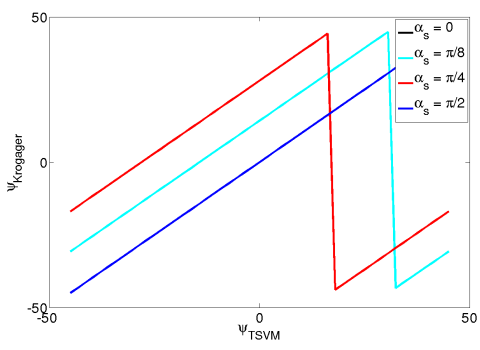

(c)

Fig. 1. Comparison between $\psi_{T S V M}$ and $\psi_{K r o g a g e r}$ : (a) as a function of $\tau_{m}$ for $\alpha_{s}=\pi / 3$ and $\Phi_{\alpha_{s}}=\pi / 3$, (b) as a function of $\Phi_{\alpha_{s}}$ for $\alpha_{s}=\pi / 3$ and $\tau_{m}=\pi / 8$ and (c) as a function of $\alpha_{s}$ for $\Phi_{\alpha_{s}}=\pi / 3$ and $\tau_{m}=\pi / 8$

square root of a positive random variable $\tau$ (representing the texture) with an independent circular complex Gaussian vector $\mathbf{z}$ with zero-mean and covariance matrix $[M]=E\left\{\mathbf{z z}^{H}\right\}$ (representing the speckle).

$$
\left\{\begin{array}{l}
H_{0}: \mathbf{k}=\mathbf{c} \\
H_{1}: \mathbf{k}=\alpha \mathbf{p}+\mathbf{c}
\end{array}\right.
$$

The optimal detector under the SIRV hypothesis is given by the following relation:

$\Lambda([M])=\frac{p_{\mathbf{k}}\left(\mathbf{k} / H_{1}\right)}{p_{\mathbf{k}}\left(\mathbf{k} / H_{0}\right)}=\frac{h_{p}\left((\mathbf{k}-\mathbf{p})^{H}[M]^{-1}(\mathbf{k}-\mathbf{p})\right)}{h_{p}\left(\mathbf{k}^{H}[M]^{-1} \mathbf{k}\right)} \underset{H_{0}}{\underset{H_{1}}{\gtrless}} \lambda$. where $h_{p}(\cdot)$ is the density generator function. Its expression is given by:

$$
h_{p}(x)=\int_{0}^{+\infty} \frac{1}{\tau^{p}} \exp \left(-\frac{x}{\tau}\right) p_{\tau}(\tau) d \tau .
$$

This optimal detector depends on the texture probability density function $p_{\tau}$.

\subsection{GLRT-LQ detector}

The Generalized Likelihood Ratio Test - Linear Quadratic (GLRT-LQ) detector can be used to detect a particular target. Let $\mathbf{p}$ be a steering vector and $\mathbf{k}$ the observed signal. The GLRT-LQ between $\mathbf{p}$ and $\mathbf{k}$ is given by [9]:

$$
\Lambda([M])=\frac{\left|\mathbf{p}^{H}[M]^{-1} \mathbf{k}\right|^{2}}{\left(\mathbf{p}^{H}[M]^{-1} \mathbf{p}\right)\left(\mathbf{k}^{H}[M]^{-1} \mathbf{k}\right)} \underset{H_{0}}{\stackrel{H_{1}}{\gtrless}} \lambda,
$$

where $[M]$ is the covariance matrix of the population under the null hypothesis $H_{0}$, i.e. the observed signal is only the clutter.

In general, the covariance matrix is unknown. One solution consists in estimating the covariance matrix $[M]$ by $[\hat{M}]_{F P}$, the fixed point covariance matrix estimator. It is the maximum likelihood estimator of the normalized covariance matrix under the deterministic texture in a Spherically Invariant Random Process. Its expression is given by the solution of the following recursive equation [10]:

$$
[\hat{M}]_{F P}=f\left([\hat{M}]_{F P}\right)=\frac{p}{N} \sum_{i=1}^{N} \frac{\mathbf{k}_{i} \mathbf{k}_{i}^{H}}{\mathbf{k}_{i}^{H}[\hat{M}]_{F P}^{-1} \mathbf{k}_{i}} .
$$

Replacing $[M]$ by $[\hat{M}]_{F P}$ in $(13)$ leads to an adaptive version of the GLRT-LQ detector.

If the covariance matrix is estimated by the fixed point estimator (14), it has been proved, for large $N$, the relation between the false alarm probability $p_{f a}$ and the detection threshold $\lambda$ :

$$
p_{f a}=(1-\lambda)^{(a-1)}{ }_{2} F_{1}(a, a-1 ; b-1 ; \lambda),
$$

with $a=\frac{p}{p+1} N-p+2$ and $b=\frac{p}{p+1} N+2 . \quad N$ is the number of pixels used to estimate the covariance matrix $[M] . p$ is the dimension of the target vector $(p=3$ for the monostatic case). ${ }_{2} F_{1}(\cdot, \cdot ; \cdot ; \cdot)$ is the Gauss hypergeometric function.

\section{DETECTION RESULTS ON A RAMSES X-BAND DATA-SET}

In this section, a real data-set acquired by the RAMSES sensor at X-band is analyzed. Fig. 2 shows a colored composition in the Pauli basis of the target vector. This data-set is 


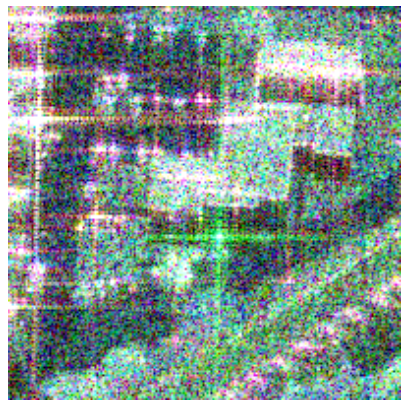

(a)

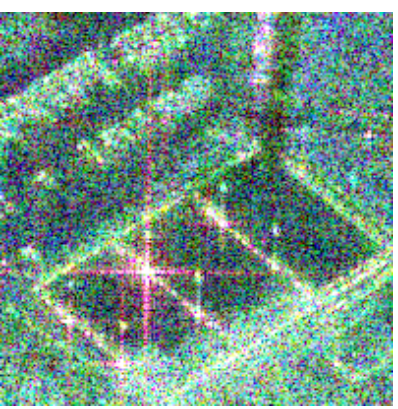

(b)
Fig. 2. Toulouse, RAMSES PolSAR data, X-band $(150 \times 150$ pixels). Colored composition in the Pauli basis of the target vector $[k]_{1}-[k]_{3}-[k]_{2}$. Images containing a dihedral (a) and a narrow diplane (b).

composed by two particular targets: a dihedral (in green on Fig. 2(a) and a narrow diplane (in red on Fig. 2(b)). Both GLRT-LQ Krogager and GLRT-LQ TSVM detectors (tilt angle estimated respectively by $\psi_{\text {Krogager }}$ and $\psi_{T S V M}$ ) are applied on this data-set. Table. 1 shows the criterion characteristics for the dihedral and the narrow diplane. As those two targets have theoretically a null target helicity $\tau_{m}$, both detectors should have similar performance. For a fixed false alarm probability of $5 \times 10^{-3}$, the detection threshold is $\lambda=0.931$. For the dihedral, The GLRT-LQ TSVM is able to detect the target $(0.956>\lambda)$ whereas the GLRT-LQ Krogager detector fails $(0.912<\lambda)$.

Similar conclusions can be done for the narrow diplane as observed on Table.1.

\begin{tabular}{||c|c|c|c|c|c||}
\hline \hline & \multicolumn{5}{|c||}{ dihedral } \\
\cline { 2 - 6 } & GLRT-LQ & $\psi$ & $\alpha_{s}$ & $\Phi_{\alpha_{s}}$ & $\tau_{m}$ \\
\hline Krogager & 0.912 & 0.761 & & & \\
\hline TSVM & 0.956 & 0.770 & -1.453 & 0.450 & -0.178 \\
\hline Pure target & \multicolumn{5}{|c||}{ narrow diplane } \\
\hline \hline & \multicolumn{6}{|c|}{} & & \\
\hline \hline & GLRT-LQ & $\psi$ & $\alpha_{s}$ & $\Phi_{\alpha_{s}}$ & $\tau_{m}$ \\
\cline { 2 - 7 } & 0.828 & -0.023 & & & \\
\hline Krogager & 0.849 & -0.026 & 1.210 & -0.172 & 0.052 \\
\hline TSVM & & & 1.249 & 0 & 0 \\
\hline Pure target & &
\end{tabular}

Table 1. Detector characteristics for the dihedral and the the narrow diplane.

\section{CONCLUSION}

In this paper, authors have proposed to the use Target Scattering Vector Model to extract the roll-invariant target vector. Some comparisons have been done between the orientation angle estimated with the phase difference between right-right and left-left circular polarizations and this issued from the TSVM. Next, authors have proposed to use the TSVM for a roll-invariant target detection. The GLRT-LQ similarity measure has been implemented and validated on high resolution
PolSAR data for the detection of particular targets such as an oriented dihedral.

Further works will deal with the use of optimal detectors based on the statistics of the PolSAR clutter. Special interest will also be dedicated to bistatic PolSAR imagery where the cross-polarization terms of the scattering matrix $\mathbf{S}$ are not equal in general [11]. In this case, two orientation angles, one at the emission and one at the reception, should be taken into account.

\section{Acknowledgment}

The authors wish to thank the French Research Agency (ANR) for supporting this work through the EFIDIR project (ANR-2007-MCDC0-04, http://www.efidir.fr).

\section{REFERENCES}

[1] R. Touzi, "Target Scattering Decomposition in Terms of RollInvariant Target Parameters," IEEE Transactions on Geoscience and Remote Sensing, vol. 45, no. 1, pp. 73-84, January 2007.

[2] E. Krogager, Aspects of Polarimetric Radar Imaging, Ph.D. thesis, Univ. Denmark, Copenhagen, Denmark, 1993.

[3] K. Kennaugh, "Effects of Type of Polarization On Echo Characteristics," Ohio State Univ., Research Foundation Columbus Antenna Lab, Tech. Rep. 389-4, 381-9, 1951.

[4] J.R. Huynen, "Measurement of the target scattering matrix," Proceedings of the IEEE, vol. 53, no. 8, pp. 936-946, August 1965.

[5] J.R. Huynen, Phenomenological Theory of Radar Targets, Academic Press, 1978.

[6] S.H. Bickel, "Some Invariant Properties of the Polarization Scattering Matrix," Proceedings of the IEEE, vol. 53, no. 8, pp. 1070-1072, August 1965.

[7] J.S. Lee, D.L. Schuler, T.L. Ainsworth, E. Krogager, D. Kasilingam, and W.-M. Boerner, "On the Estimation of Radar Polarization Orientation Shifts Induced by Terrain Slopes," IEEE Transactions on Geoscience and Remote Sensing, vol. 40, no. 1, pp. 30-41, January 2002.

[8] L. Bombrun, G. Vasile, M. Gay, J.-P. Ovarlez, and F. Pascal, "A combined TSVM model and GLRT detector for a roll invariant target detection," in European Conference on Synthetic Aperture Radar, EUSAR'10, Aachen, Germany, 2010.

[9] E. Conte, M. Lops, and G. Ricci, "Adaptive Radar Detection in Compound-Gaussian Clutter," in Proc. of the European Signal processing Conf., Edinburgh, Scotland, September 1994.

[10] F. Gini and M. V. Greco, "Covariance matrix estimation for CFAR detection in correlated heavy tailed clutter," Signal Processing, vol. 82, no. 12, pp. 1847-1859, 2002.

[11] L. Bombrun, "Extension of the Target Scattering Vector Model to the Bistatic Case," in Geoscience and Remote Sensing, IGARSS'10, Hawaii, USA, 2010. 